
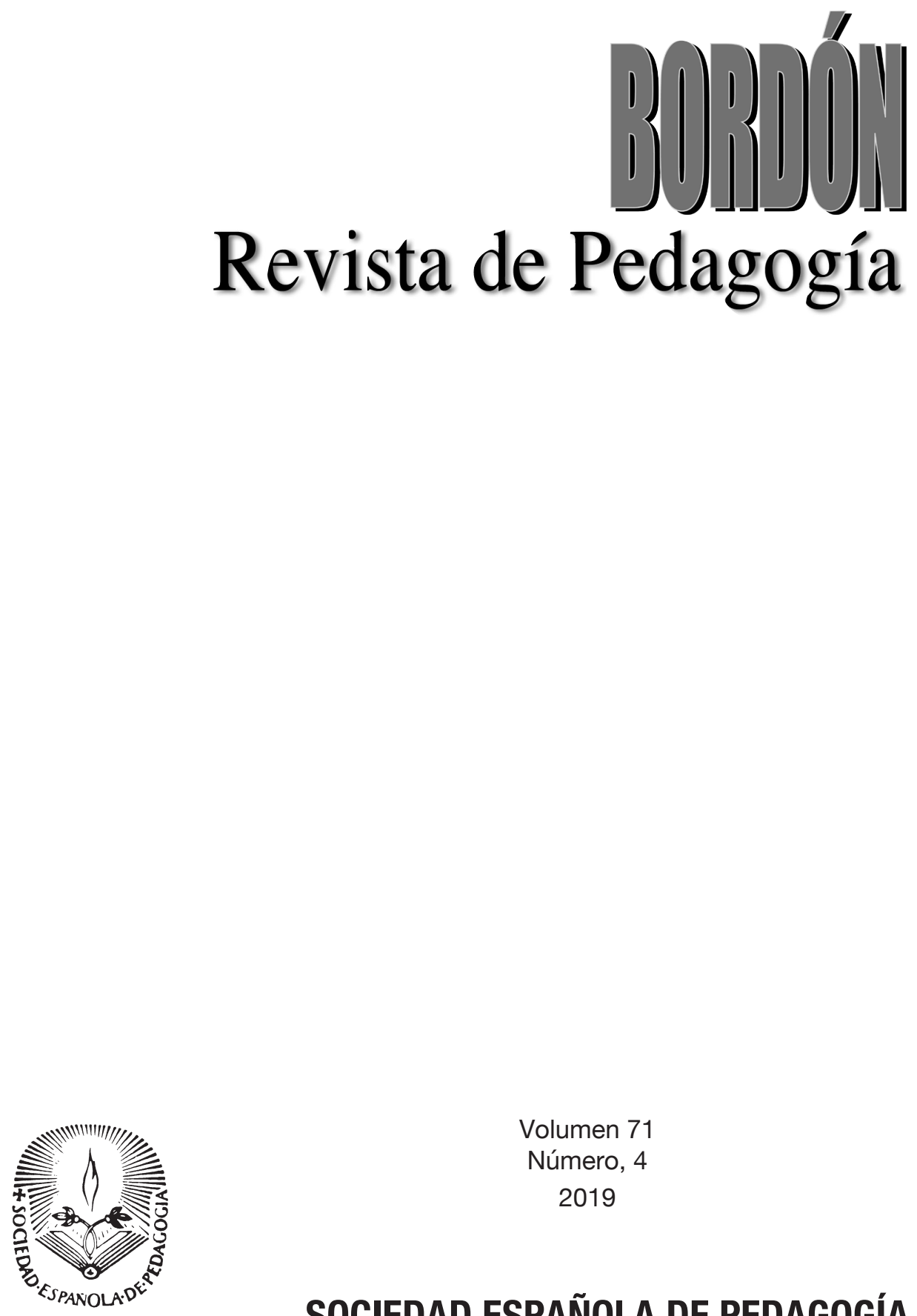

Volumen 71

Número, 4

2019 


\title{
LA EDUCACIÓn SECUNDARIA EN CINCO PAÍSES DE AMÉRICA DEL SUR: DEFInICIONES NORMATIUAS Y ALCAICES EMPÍRICOS DESDE LA PERSPECTIUA DEL DERECHO A LA EDUCACIÓn
} Secondary Education in five South American countries: normative
definitions and empirical results from the right to education
perspective

\author{
GUILLERMO RAMÓN RUIZ(1), SEBASTIÁN SCIOSCIOLI(2) Y MIRIAM LORENTE RODRÍGUEZ(3) \\ (1) Universidad de Buenos Aires, Consejo Nacional de Investigaciones Científicas y Técnicas (Argentina) \\ (2) Universidad de Buenos Aires (Argentina) \\ (3) Universidad de Valencia (España)
}

D01: 10.13042/Bordon.2019.69459

Fecha de recepción: 22/12/2018 - Fecha de aceptación: II/10/2019

Autor de contacto / Corresponding author: Guillermo Ramón Ruiz. E-mail: gruiz@ derecho.uba.ar

INTRODUCCIÓN. En América Latina muchas reformas educativas han avanzado en definiciones normativas sobre el derecho a la educación. Es posible pensar que dichas reformas obedecen al impacto del derecho internacional de los derechos humanos hacia el interior de los derechos nacionales. En cinco países de América del Sur se presentó a la ampliación de los años de educación escolar obligatoria como una política que forma parte del contenido del derecho a la educación. MÉTODO. En términos metodológicos, el diseño de esta investigación incluye técnicas cualitativas entre las que destacan el análisis jurídico normativo y la consecuente generación de tipificaciones comparativas de las fuentes provenientes de los países considerados. También se utilizaron técnicas cuantitativas para el análisis de estadísticas educativas para poder dimensionar la cobertura educativa a la luz de las inversiones presupuestarias realizadas en cada Estado. RESULTADOS. En estos países las reformas han fijado un nuevo rango de estudios obligatorios que incluye toda la educación secundaria. Entre los argumentos esgrimidos se encuentran la atención a las demandas educativas de los sectores que no se encontraban escolarizados; con ello se favorecería el logro de una ciudadanía más efectiva en las jóvenes generaciones. DISCUSIÓN. Se revisan críticamente los conceptos de "derecho a la educación" y de "obligatoriedad escolar" para cuestionar las reformas recientes de cinco países de América del Sur que han establecido la obligatoriedad de la escuela secundaria. La comparación transnacional se centra en las políticas de reforma desde el enfoque de los derechos humanos. Cuestiones tales como la extensión de la obligatoriedad, la cobertura y la inversión educativa son puestas bajo análisis.

Palabras clave: Derecho a la educación, Escuela secundaria, Reformas educativas, América del Sur. 


\section{Introducción y encuadre metodológico}

El derecho a la educación y la obligatoriedad escolar constituyen términos (para las ciencias jurídicas y las ciencias de la educación) que suelen estar vinculados en diferentes ámbitos políticos y académicos. Así, la extensión de la obligatoriedad escolar hasta completar toda la educación secundaria ha sido una de las políticas que algunos Estados latinoamericanos han llevado a cabo durante las últimas décadas como forma de atender las demandas educativas y favorecer el logro de una ciudadanía más efectiva. Ello permitiría comprender algunas razones por las cuales muchas reformas escolares han avanzado en definiciones normativas sobre el derecho a la educación.

En contextos de reformas educativas, cabría prestar atención a cómo inciden las nuevas realidades espaciales en las transferencias internacionales de discursos y políticas de reforma. Dichas realidades espaciales mantienen pero también desbordan al Estado-nación como eje analítico central para el estudio de la transferencia. Las dimensiones espaciales en las cuales operan las políticas educativas revelan nuevos modos en los que se gobierna la educación formal y su organización en contextos geográficos con múltiples movilidades educativas (Schriewer, 2014; Steiner-Khamsi, 2010). Algunos autores advierten que para responder a los contradictorios procesos globalizadores es preciso reconocer que el uso del concepto de Estado-nación debe ser considerado "como el explanandum, como necesidad de explicación más que como explanans, como el para qué de la explicación (Roberston y Dale, 2012: 112). De este modo, cabría considerar a la educación formal como una educación constituida a la luz de complejos mecanismos de las redivisiones funcionales y escalares (internacionales, regionales, multilaterales) de la gobernanza educativa, así como también a partir de agentes locales que se interpretan en función de las coyunturas nacionales específicas.
En este artículo nos concentraremos en la revisión de algunas de las recientes reformas de cinco países de América del Sur que han establecido la obligatoriedad de la escuela secundaria como forma de garantizar un ejercicio efectivo del derecho a la educación. Para ello realizamos un abordaje en el cual la comparación transnacional se centra en las políticas de reforma de nivel secundario a la luz del enfoque de derechos humanos, y toma el concepto de "derecho a la educación" como dimensión de análisis principal para analizar los procesos de reformas que algunos países de América del Sur han llevado a cabo. En la selección de los casos hemos considerado que un criterio de demarcación podría deberse a alguna diferenciación regional, política e institucional que exista entre los países sudamericanos. En tal sentido, entendemos que los casos de algunos países que integran el Mercado Común del Sur (MERCOSUR) ${ }^{1}$ pueden tomarse como objeto de nuestro análisis comparativo regional. Incluso en estos países se observa que el desarrollo y las dimensiones de sus sistemas educativos reflejan características diversas y diferenciales en sus estructuras sociales. Las marcas históricas, así como las definiciones contemporáneas, se asientan sobre sistemas escolares muy distintos en lo que atañe a su organización institucional y académica, lo cual redunda en adaptaciones particulares de los discursos de reformas educativas que circulan en la región y que los países incluyen en sus procesos y políticas educativas. Así, de acuerdo con las aportaciones de Cais (2002), se realiza un análisis entre países (cross-national) a través de la estrategia de análisis de casos y se emplea la comparación de sistemas similares (en aspectos culturales, históricos o económicos). En primer lugar, nos centramos en las definiciones conceptuales del encuadre teórico y de los conceptos analizados: derecho a la educación y obligatoriedad escolar. En segundo lugar, se presentan las políticas educativas de reformas escolares que consideran a la educación obligatoria como objeto de análisis desde un enfoque de derechos. En tercer lugar, consideramos los alcances que han tenido dichos políticas a la luz 
de algunos indicadores regionales sobre la expansión y cobertura escolar, así como la inversión realizada por los países estudiados. Finalmente, discutimos dichas políticas en función de las transferencias de discursos de reformas que han promovido la Educación Secundaria Obligatoria.

\section{Definiciones conceptuales}

\section{La educación como derecho fundamental}

Reconocer la educación como derecho humano fundamental implica un cambio radical en el modo en que se concibe la relación entre sujetos activos de la educación (especialmente niños/as y adolescentes) y el Estado. Esta mirada implica avanzar hacia el estudio de la dinámica educativa desde un enfoque de derechos, desde el cual la labor del Estado en la provisión de servicios básicos implica un desempeño esencial de construcción de ciudadanía. Aquí adquiere relevancia el principio de ciudadanía, a partir del cual la titularidad de los derechos de los habitantes guía la política pública en función del marco normativo de los derechos humanos plasmado en normas tanto nacionales como internacionales (Scioscioli, 2014). El énfasis en la concepción y estructura del derecho como derecho fundamental implica el reconocimiento de posiciones jurídicas que deben ser respetadas tanto por particulares como por el Estado, por medio de acciones positivas y omisiones $^{2}$. La reconstrucción de estas posiciones se traduce para el Estado en las obligaciones de proteger, organizar, coordinar, disponer recursos, fiscalizar el sistema educativo garantizando al individuo que frente al incumplimiento de las obligaciones pueda exigir su cumplimiento por la vía administrativa o judicial.

De acuerdo con el derecho internacional de los derechos humanos, el derecho a la educación comprende desde el plexo normativo internacional (y también local) un importante catálogo de obligaciones a cargo de los Estados nacionales.
Estas obligaciones, en función del contenido de este derecho, pueden clasificarse como:

- Obligación de respetar: esta obligación se observa cuando el derecho a la educación se comporta justamente como derecho de prestación negativa o no intervención.

- Obligación de proteger: esta obligación exige a los Estados adoptar leyes u otras medidas que resulten necesarias para evitar o prevenir que los particulares produzcan dichos perjuicios.

- Obligación de cumplir, realizar y garantizar: este aglomerado es el más interesante en función del contenido social del derecho a la educación y de cara al contexto de exclusión social que afecta a los países aquí estudiados. Esta obligación se define como la necesidad de que los Estados reconozcan este derecho en sus ordenamientos jurídicos y que adopten una política nacional acompañada de un plan detallado para el ejercicio del derecho.

En educación, el catálogo de posiciones jurídicas garantizadas ha sido desarrollado en el artículo 13 del Pacto Internacional de Derechos Económicos, Sociales y Culturales, que ha sido objeto de una exégesis tanto por parte del Comité de Derechos Económicos, Sociales y Culturales (Observación General N. ${ }^{\circ} 13$ ) como por parte del desarrollo analítico de la Relatora Especial de Naciones Unidas sobre el derecho a la educación (Tomasevsky, 2001). La obligatoriedad escolar secundaria, entendida a priori como la extensión de los estudios generales de nivel posprimario, accesible para todas las personas, toca transversalmente todos los aspectos que estructuran el contenido del derecho a la educación como derecho humano y redefinen también el peso de las obligaciones de los Estados nacionales para con este derecho. El contexto latinoamericano tiene mucho que aportar dadas las reformas normativas que apuntaron a reconocer las condiciones materiales de vigencia del 
derecho a la educación, así como la adopción de nuevas cláusulas constitucionales que reconocieran la legitimidad del reclamo hacia el Estado a efectos de que este tenga un rol más activo como garante de los contenidos presentes en dicho derecho (Gargarella y Courtis, 2009). En este esquema, la extensión de la obligatoriedad escolar se esgrime como uno de los instrumentos más concretos para combatir las desigualdades fácticas (de redistribución y simbólicas) de la sociedad y que afectan particularmente a sus grupos más vulnerables.

\section{La obligatoriedad escolar en el marco de las políticas de reformas}

La obligatoriedad escolar evidenció durante los últimos ciento cincuenta años una clara expansión, con su prolongación hacia edades más tempranas y también hacia edades más avanzadas de la vida de las personas. Esa ampliación se debió tanto a las políticas educativas orquestadas por los Estados nacionales como a los instrumentos internacionales de derecho que han regulado los alcances y las implicaciones del derecho a la educación, así como a las obligaciones del Estado para garantizar el ejercicio de ese derecho a los habitantes de su territorio. Diversos organismos internacionales, en la medida en que han incidido cada vez más en la definición del debate político pedagógico contemporáneo, han promovido reformas educativas en las últimas décadas que incluyen la extensión de los estudios obligatorios.

Ahora bien, en la medida en que se volvió obligatoria, la educación secundaria pasó a conformar una educación general de nivel posprimario. No obstante, si la educación secundaria también es el nivel previo a la superior y se le exigen niveles de formación y carácter propedéutico-académicos, requiere niveles de exigencia orientados a satisfacer la formación ulterior. He aquí uno de los mayores dilemas pedagógicos que afrontan los desarrollos actuales de los sistemas educativos. Este dilema es parte - a la vez- de la difusión de procesos de reformas que promueven transformaciones sobre la escolarización masiva. Desde nuestra perspectiva consideramos que la transferencia —recepción y traducción- de discursos y los proyectos político-educativos, presentes desde el siglo XIX, cobran nuevas formas en la actualidad: el complejo y multidimensional proceso denominado en inglés borrowing and lending y la adaptación de políticas educativas incluyen nuevos agentes además de los Estados-Nación (Steiner-Khamsi, 2010). Esta reconceptualización permite interpretar críticamente los alcances de los recurrentes procesos de reformas educativas del nivel secundario.

\section{Resultados: las reformas en la educación secundaria}

El encuadre conceptual precedente nos permite analizar la situación de los países aquí considerados, dado que a partir de la década de los noventa estos han implementado diferentes reformas estructurales de sus sistemas educativos que han extendido la obligatoriedad de los estudios posprimarios. Las transformaciones han estado encuadradas en procesos de reformas de los Estados que han respondido a presiones internacionales de organismos financieros, los cuales han propiciado el ajuste del costo de los aparatos de los Estados como forma de resolver crisis económicas y sociales. Dichos procesos de ajuste han derivado en resultados muy disímiles, pero en casi todos críticos sobre las formas de regulación y financiamiento de la educación pública, a la par que han favorecido procesos de descentralización educativa y de nuevas formas de relación con el sector privado de la educación. Las reformas iniciadas con el nuevo siglo han modificado a su vez algunas de las orientaciones de aquellas instrumentadas en la década previa, pero han vuelto a modificar la estructura académica y los rangos de obligatoriedad de los estudios escolares como parte de transferencias internacionales de discursos para promover la igualdad educativa. Las reformas contemporáneas se asientan sobre sistemas muy distintos. 
TABla 1. Organización institucional y estructura académica de los sistemas escolares de cinco países de América del Sur

\begin{tabular}{|c|c|c|c|c|c|}
\hline Indicador/ País & Argentina & Bolivia & Brasil & Paraguay & Uruguay \\
\hline $\begin{array}{l}\text { Régimen de } \\
\text { gobierno }\end{array}$ & Federal & $\begin{array}{l}\text { Régimen } \\
\text { centralizado } \\
\text { con niveles de } \\
\text { descentralización } \\
\text { y autonomías } \\
\text { (Estado } \\
\text { plurinacional) }\end{array}$ & Federal & $\begin{array}{l}\text { Régimen } \\
\text { centralizado }\end{array}$ & $\begin{array}{l}\text { Régimen } \\
\text { centralizado }\end{array}$ \\
\hline $\begin{array}{l}\text { Organización } \\
\text { política }\end{array}$ & $\begin{array}{l}23 \text { provincias } \\
\text { y } 1 \text { ciudad } \\
\text { autónoma }\end{array}$ & $\begin{array}{l}9 \\
\text { departamentos }\end{array}$ & $\begin{array}{l}26 \text { Estados, } \\
5.564 \text { municipios } \\
\text { y el Distrito } \\
\text { Federal }\end{array}$ & $\begin{array}{l}17 \\
\text { departamentos }\end{array}$ & $\begin{array}{l}19 \\
\text { departamentos }\end{array}$ \\
\hline Población & 43,4 millones & 10,72 millones & 207,8 millones & 6,7 millones & 3,4 millones \\
\hline $\begin{array}{l}\text { Población } \\
\text { escolar } \\
\end{array}$ & 13.004 .334 & 3.005 .841 & 60.693 .813 & 2.278 .803 & 990.998 \\
\hline $\begin{array}{l}\text { Rango de } \\
\text { obligatoriedad }\end{array}$ & 14 años & 14 años & 14 años & 14 años & 14 años \\
\hline $\begin{array}{l}\text { Reforma } \\
\text { educativa }\end{array}$ & $\begin{array}{l}\text { Año } 2006 . \\
\text { Ley de } \\
\text { Educación } \\
\text { Nacional N. } \\
26.206\end{array}$ & $\begin{array}{l}\text { Año } 2010 . \\
\text { Ley de la } \\
\text { Educación } \\
\text { Avelino Siñani- } \\
\text { Elizardo Pérez } \\
\text { N. }{ }^{\circ} 70\end{array}$ & $\begin{array}{l}\text { Año } 1996 . \\
\text { Ley de } \\
\text { Directrices } \\
\text { y Bases de la } \\
\text { Educación } \\
\text { Nacional } \\
\text { N. }{ }^{\circ} 9.394\end{array}$ & $\begin{array}{l}\text { Año } 1998 . \\
\text { Ley General de } \\
\text { Educación N.º }^{\circ} \\
1.264\end{array}$ & $\begin{array}{l}\text { Año } 2008 . \\
\text { Ley de Educación } \\
\text { N. }{ }^{\circ} 18.437\end{array}$ \\
\hline
\end{tabular}

Fuente: elaboración propia a partir de las normativas educativas y datos demográficos de cada país.

La tabla precedente da cuenta de las diferentes formas organizativas de los sistemas escolares, derivadas de los regímenes de gobierno de cada uno de los Estados considerados. Las diferencias se verifican también en las dimensiones de cada sistema educativo, que refleja la estructura social e institucional de estos países. Algunas de las leyes de reforma fueron aprobadas en la década de los noventa (Brasil y Paraguay) y tuvieron modificaciones ulteriores, además, están las aprobadas entre los años 2006 y 2010 (Argentina, Uruguay y Bolivia). Si se atiende a la estructura académica prevista en estos marcos normativos, se evidencian ciertas semejanzas en cuanto a la organización de los niveles, aunque con una clara diferenciación en el caso de
Bolivia al contemplar diferentes subsistemas, entre los cuales se ubica la educación formal. En los casos considerados, la extensión de la obligatoriedad alcanza a toda la educación secundaria, lo que es destacable a la luz de nuestro planteamiento en relación con el complejo y multidimensional proceso de transferencias internacionales de reformas que promueven la obligatoriedad de la educación secundaria como parte de las obligaciones de los Estados para garantizar el derecho a la educación. Más allá de las definiciones normativas, cabe analizar algunos de los efectos de estas políticas de reformas en cuanto a los indicadores del rendimiento interno de los sistemas escolares y de las inversiones realizadas. 


\section{Discusión}

Indicadores en relación con los logros educativos

La evolución y expansión de la escolarización ha permitido plantearse la finalización del nivel secundario de educación como una meta $y$, en consecuencia, su obligatoriedad como un deber de los Estados en relación con el respeto, protección, realización, cumplimiento y garantía del derecho a la educación. Todo en función de los beneficios de la educación formal (Tedesco, 2012; Vila, 2003). Asimismo, constituye la premisa fundante que se encuentra en la base de las agendas mundiales de educación (entre ellas las Metas educativas 2021 y la Agenda de educación 2030, ODS) ${ }^{3}$. Las siguientes tablas permiten evidenciar la evolución reciente de algunos indicadores.

En el análisis global de las tablas 2, 3 y 4 puede apreciarse que la educación secundaria muestra un panorama aún más complejo que la educación

TABla 2. Tasas netas de matrícula en educación primaria y educación secundaria, según país y año. América del Sur (países seleccionados)

\begin{tabular}{lcccc} 
& \multicolumn{4}{c}{ Acceso } \\
\cline { 2 - 3 } País/Indicador & \multicolumn{2}{c}{ Tasa neta de matrícula en primaria } & & Tasa neta de matrícula en secundaria \\
\cline { 2 - 3 } \cline { 5 - 5 } & 2007 & 2016 & 2007 & 2016 \\
\hline Argentina & 99,1 & 99,0 & 79,2 & 89,5 \\
\hline Bolivia $^{1}$ & 95,4 & 91,9 & 73,5 & 82,3 \\
\hline Brasil & 91,6 & 78,0 & 73,2 & 64,9 \\
\hline Paraguay & 91,3 & 98,0 & 58,8 & 82,8 \\
\hline Uruguay & 97,8 & & 67,6 & \\
\hline
\end{tabular}

Fuente: elaboración propia a partir de la base de datos de la CEPAL.

Nota: ${ }^{1}$ Los datos de Bolivia para el último año disponible pertenecen a 2017.

TABla 3. Tasas de eficiencia interna y permanencia en la educación secundaria para el último año disponible, según país y año. América del Sur (países seleccionados)

Eficiencia interna y permanencia

País/Indicador

$\begin{gathered}\text { Alumnos con dos o más años de retraso } \\ \text { escolar en secundaria }(\%)\end{gathered}$
$2015^{1}$

Tasa de jóvenes fuera de la escuela en edad de asistir al nivel secundario superior

2016

\begin{tabular}{lll} 
Argentina & 32,6 & 8,9 \\
\hline Bolivia & 15,5 & 17,6 \\
\hline Brasil & 26,6 & 17,2 \\
\hline Paraguay & 18,5 & 30,9 \\
\hline Uruguay & 30,2 & 19,6
\end{tabular}

Fuente: elaboración propia a partir de las bases de datos del SITEAL y de UNESCO-(UIS).

Notas: ${ }^{1}$ Los datos de Brasil y Uruguay pertenecen a 2015; los de Argentina, Bolivia y Paraguay al año 2014. ${ }^{2}$ Los datos de Bolivia pertenecen al año 2017. 
Tabla 4. Jóvenes y adultos con nivel primerio y nivel secundario completo, según país y año. Cinco países de América del Sur

\begin{tabular}{lcc} 
& \multicolumn{2}{c}{ Conclusión } \\
\cline { 2 - 3 } País/Indicador & \begin{tabular}{c} 
Personas de $\begin{array}{c}\text { 15 a 19 años con primaria } \\
\text { completa (\%) }\end{array}$ \\
\cline { 2 - 3 }
\end{tabular} & $\begin{array}{c}\text { Personas de 20 a 24 años con secundaria } \\
\text { completa (\%) }\end{array}$ \\
\hline & 9014 & 2014 \\
\hline Argentina $^{1}$ & 98,4 & 65,6 \\
\hline Bolivia $^{2}$ & 95,3 & 65,6 \\
\hline Brasil & 96,2 & 79,1 \\
\hline Paraguay & 93,7 & 61,9 \\
\hline
\end{tabular}

Fuente: elaboración propia a partir de las bases de datos del Banco Mundial, de la CEPAL y del SITEAL.

Notas: ${ }^{1}$ Los datos de conclusión de la educación primaria y secundaria pertenecen al área urbana. ${ }^{2}$ Los datos de Bolivia pertenecen al año 2013.

primaria dado que los países caminan a distintas velocidades hacia la conclusión del nivel. El acceso a la educación secundaria y su finalización distan mucho de las alentadoras cifras que ofrece la educación primaria en los mismos países aquí analizados. En este nivel educativo, la variabilidad y dispersión de los datos aumenta considerablemente en los resultados de los distintos países, pero sobre todo en los datos desagregados en función del nivel de ingresos y el área geográfica de residencia. La tasa neta de matrícula en secundaria es bastante inferior que la del nivel primario, aun cuando la secundaria forma parte de la enseñanza obligatoria, lo que da buena cuenta de los elevados niveles de fracaso y abandono escolar y, en cierta medida, de la incapacidad del sistema educativo y de las políticas estatales para retener a los estudiantes hasta el último grado de la secundaria. Retomaremos estos datos sintéticamente más adelante.

\section{Una visión panorámica de la inversión educativa}

La inversión educativa que los distintos países realizan en educación es un claro indicador del compromiso gubernamental con el desempeño de sus obligaciones en términos de cumplimiento y garantía del derecho a la educación en relación con la prestación del servicio. Así pues, se hace necesario complementar el presente estudio con el análisis general de la financiación educativa con el objetivo de ofrecer determinadas claves que inviten a la reflexión sobre la coherencia de las declaraciones y políticas nacionales con los montos invertidos y los resultados obtenidos. En la siguiente tabla se ofrece una visión comparativa y sintética de la inversión educativa a la luz de diversos indicadores.

Un indicador relevante a este respecto es el gasto del gobierno en educación calculado como porcentaje del PIB. Puede apreciarse que Bolivia es el país que mayor porcentaje de su riqueza destina a la educación con un 7,3\%, seguido de Brasil. De hecho, Bolivia es el país que mayor proporción del PIB invierte en educación de América Latina (sin contar a los países del Caribe). En una posición intermedia se encuentra Argentina con un 5,6\%, y con unos porcentajes más discretos cierran esta ordenación Uruguay y Paraguay (4,5\% y $4,6 \%$, respectivamente).

No obstante, en términos absolutos, la inversión educativa de países como Brasil o Argentina 
supera extensamente a la del resto de países estudiados en todos los niveles educativos, aspecto que también puede evidenciarse en el gasto por alumno, cuya estructura de reparto es disímil entre los distintos niveles educativos en cada país. Aun cuando la tendencia general es el aumento

Tabla 5. Inversión educativa en América del Sur (países seleccionados) ${ }^{4}$

\begin{tabular}{|c|c|c|c|c|c|c|c|}
\hline \multirow[t]{2}{*}{ País/Indicador } & \multirow{2}{*}{$\begin{array}{c}\% \text { PIB del } \\
\text { gobierno } \\
\text { destinado } \\
\text { a } \\
\text { educación }\end{array}$} & \multirow{2}{*}{$\begin{array}{c}\text { Gasto por } \\
\text { alumno } \\
\text { en } \\
\text { dólares } \\
\text { (PPP\$) } \\
\text { y nivel } \\
\text { educativo }\end{array}$} & \multirow{2}{*}{$\begin{array}{c}\text { Gasto en } \\
\text { educación } \\
\text { como \% } \\
\text { del gasto } \\
\text { total } \\
\text { del } \\
\text { gobierno }\end{array}$} & \multicolumn{2}{|c|}{$\begin{array}{c}\text { \% Gasto en secundaria } \\
\text { como \% del gasto } \\
\text { gubernamental en } \\
\text { educación }\end{array}$} & \multirow{2}{*}{$\begin{array}{c}\text { Gasto } \\
\text { público y } \\
\text { privado } \\
\text { en } \\
\text { instituciones } \\
\text { educativas }\end{array}$} & \multirow{2}{*}{$\begin{array}{c}\text { Gasto } \\
\text { promedio } \\
\text { del hogar en } \\
\text { educación } \\
\text { como \% } \\
\text { del gasto } \\
\text { total de los } \\
\text { hogares }\end{array}$} \\
\hline & & & & Total & $\begin{array}{l}\text { Secundaria } \\
\text { superior }\end{array}$ & & \\
\hline Argentina & $\begin{array}{c}5,6 \\
(2016)\end{array}$ & $\begin{array}{l}\text { P: } 3.083,68 \\
\text { S: } 4.374,00 \\
\text { T: } 3.258,20 \\
\quad(2016) \\
\end{array}$ & $\begin{array}{c}13,6 \\
(2016)\end{array}$ & $40,94(2016)$ & $\begin{array}{c}16,8 \\
(2016)\end{array}$ & $\begin{array}{l}\text { Público: } 87 \\
\text { Privado: } 13 \\
\text { (2014) }\end{array}$ & $\begin{array}{c}3,3^{5} \\
(2012)\end{array}$ \\
\hline Bolivia & $\begin{array}{c}7,3 \\
(2014)\end{array}$ & $\begin{array}{l}\text { P: } 1.564,68 \\
\text { S: } 1.206,05 \\
\text { T: ND } \\
\quad(2014)\end{array}$ & $\begin{array}{c}16,9 \\
(2014)\end{array}$ & 27,09 (2014) & $\begin{array}{c}16,3 \\
(2014)\end{array}$ & $\begin{array}{l}\text { Público: } 66,7 \\
\text { Privado: } 33,3 \\
\text { (2005) }\end{array}$ & $\begin{array}{c}6,7 \\
(2011)\end{array}$ \\
\hline Brasil & $\begin{array}{c}6,2 \\
(2015)\end{array}$ & $\begin{array}{l}\text { P: } 3.162,74 \\
\text { S: } 3.394,82 \\
\text { T: } 5.211,04 \\
\quad(2015)\end{array}$ & $\begin{array}{c}16,2 \\
(2015)\end{array}$ & $41,37(2015)$ & $\begin{array}{c}18,4 \\
(2015)\end{array}$ & $\begin{array}{l}\text { Público: } 79,7 \\
\text { Privado: } 20,3^{4} \\
\quad \text { (2007) }\end{array}$ & $\begin{array}{c}1,6^{6} \\
(2008-2009)\end{array}$ \\
\hline Paraguay & $\begin{array}{c}4,5 \\
(2016)\end{array}$ & $\begin{array}{l}\text { P: } 1.444,16 \\
\text { S: } 1.481,30 \\
\text { T: } 1.375,48^{2} \\
\quad(2016)\end{array}$ & $\begin{array}{c}18,2 \\
(2016)\end{array}$ & 31,28 (2016) & $\begin{array}{c}17,1 \\
(2016)\end{array}$ & $\begin{array}{l}\text { Público: } 59,8 \\
\text { Privado: } 40,2 \\
\text { (2010) }\end{array}$ & 4,5 (2010) \\
\hline Uruguay & $\begin{array}{c}4,6 \\
(2015)\end{array}$ & $\begin{array}{l}\text { P: } 1.060,05 \\
\text { S: } 1.259,20 \\
\text { T: } 5.248,52^{3} \\
\quad(2006)\end{array}$ & $\begin{array}{c}14,1 \\
(2012)\end{array}$ & 33,17 (2011) & $\begin{array}{c}15,1 \\
(2011)\end{array}$ & $\begin{array}{l}\text { Público: } 74,4 \\
\text { Privado: } 25,6 \\
\text { (2015) }\end{array}$ & $\begin{array}{c}3,2^{7} \\
(2015)\end{array}$ \\
\hline
\end{tabular}

Fuente: elaboración propia a partir de UIS.Stat (2018); Acerenza, S. y Gandelman, N. (2017); Bottinelli, L. y Sleiman, C. (2014); INEEd (2017); OECD (2017); OEI (2016); UDAPE/UNICEF (2008); Menezes-Filho, N. A. y Núñez, D. F. (2012); Urquiola, M. (2011).

Notas: ${ }^{1}$ financiamiento gubernamental de la educación por estudiante (excluyendo las transferencias internacionales al gobierno para educación). Las equivalencias son $\mathrm{P}=$ primaria, $S=$ secundaria, $\mathrm{T}=$ terciaria, $\mathrm{ND}=$ no disponible. ${ }^{2}$ El dato para la educación terciaria pertenece al año 2010. ${ }^{3}$ El dato para la educación terciaria pertenece al año 2011. ${ }^{4}$ La tasa de gasto privado/PIB se refiere a la tasa calculada a través de Pesquisa de Orçamentos Familiares (POF) 2008/2009. ${ }^{5}$ Hace referencia al total del área urbana. ${ }^{6}$ El dato representa solamente el gasto en educación formal, por lo que se estima será ligeramente superior al presentado. ${ }^{7}$ El dato considera los pagos por concepto de matrículas, cuotas y exámenes en instituciones educativas. Excluye otros gastos relevantes como materiales, libros de texto, transporte para asistir a los centros, vestimenta, campamentos o actividades recreativas vinculadas a la finalización de cursos, por lo que se estima será ligeramente superior al presentado. 
del gasto educativo a medida que aumenta el nivel educativo, no siempre se cumple en los países analizados. Este es el caso de Argentina y Paraguay, donde la inversión en educación secundaria es mayor que la realizada en el nivel terciario.

A su vez, el porcentaje que los Estados invierten en educación como porcentaje del gasto total del gobierno ayuda a situar la relevancia que tiene este apartado dentro del conjunto de sectores en los que invierten en un determinado año. A este respecto, Paraguay es el país que dedica mayor porcentaje del gasto total, seguido de Bolivia y Brasil. Argentina se sitúa en el extremo contrario con un porcentaje del $13,6 \%$, más de 3 puntos porcentuales por debajo del promedio regional. Aunque Paraguay se sitúa en la parte alta respecto a este indicador, lo cierto es que el Estado ha reducido la inversión por debajo del mínimo presupuestario en los últimos años (2014-2016). En este sentido, Molinier (2016) explica que este hecho está vinculado con la reducción del gasto ejecutado en los programas de acción (particularmente en los relacionados con los niveles de la educación obligatoria) y de inversión.

Por su parte, el INEEd (2017) plantea la especial relevancia que tiene en el caso uruguayo la estructura poblacional en la relativización de la interpretación de que un menor gasto en educación con respecto al PIB o como porcentaje del gasto total en comparación con otros países latinoamericanos supone un menor esfuerzo por parte del gobierno, dado que esta es más similar a la de los países desarrollados con poblaciones más envejecidas donde el número de nacimientos se ha reducido considerablemente y, en consecuencia, desde el año 2005 también lo ha hecho la población en edad de asistir al sistema escolar. Si bien es cierto, todavía distan de alcanzar una inversión en educación del 6\% del PIB cuya consecución ha sido un referente en la política educativa en el país.

Concretamente, si nos centramos en el esfuerzo inversor en el tramo secundario de educación, que es el considerado en este estudio, puede advertirse que es prioritario en países como Argentina y Brasil, donde recibe la mayor parte de la inversión económica por parte del Estado en comparación con el resto de niveles educativos. Paraguay y Uruguay se encuentran en una posición intermedia con una distribución más equitativa entre el nivel primario, secundario y terciario. Además, en el caso uruguayo la educación secundaria es también la que recibe el mayor monto económico. En el caso boliviano, la prioridad es el nivel primario con porcentajes similares a los establecidos en Argentina y Brasil para el nivel secundario, mientras que la educación secundaria y superior mantienen porcentajes similares en torno al $26 \%$ y $27 \%$.

Asimismo, la inversión de los distintos países en el tramo de la educación superior se mantiene entre el 15\% del gasto total en educación en Uruguay y el 18,4\% en Brasil. La variabilidad en este indicador es de apenas un 3\%. Los niveles de acceso y eficacia interna de la educación secundaria, en concreto de la educación superior en ambos países (que se sitúan en el extremo inferior y superior), pueden ayudar al correcto análisis de este indicador relativizando, en cierta medida, la correspondencia entre un menor porcentaje de gasto en el país uruguayo y una menor implicación o esfuerzo del gobierno con este nivel educativo. No obstante, si se analiza la evolución de este indicador en cada uno de los países a lo largo de los años puede advertirse una tendencia negativa en Uruguay desde el año 2005 hasta el presente (descendió cerca de 2 puntos porcentuales).

Así pues, de los países aquí seleccionados, solo en Uruguay, Bolivia y Paraguay la distribución del gasto entre la escolarización secundaria superior e inferior es similar para ambos niveles y ligeramente favorable para el último tramo de la secundaria en el caso de Bolivia y Paraguay. A este respecto, variables como las tasas brutas de matrícula, la complejidad (modalidades, opciones) o la duración de uno u otro periodo de la escolaridad secundaria ayudarían a revelar 
algunas claves de este reparto, así como ciertas discordancias. Sin adentrarnos en profundidad en este análisis que supondría una amplia disertación per se, es necesario destacar el caso boliviano, en el que inicialmente resulta incoherente un mayor esfuerzo inversor en el nivel secundario superior por parte del Estado cuando la provisión en la secundaria inferior se estima más elevada al duplicar los años de escolaridad oficial de este tramo al de la secundaria superior (cuatro años frente a dos años) y ser la tasa de matrícula bruta más elevada.

En cuanto al componente público y privado de la inversión educativa, es importante señalar el alto componente público de Argentina. De hecho, Bottinelli y Sleiman (2014) afirman que en Argentina históricamente destaca una importante contribución del Estado en la provisión del servicio educativo. Le siguen Brasil y Uruguay, donde el componente privado supone más de una quinta y cuarta parte del gasto en educación, respectivamente (20,3\% y 25,4\%). Estos datos sitúan a ambos países cercanos al promedio iberoamericano, aunque sin superarlo $(26,2 \%)^{5}$. Si bien es cierto, al compararlo con el promedio de los países de la Organización para la Cooperación y el Desarrollo Económicos (OCDE), el componente privado de la educación resulta ciertamente elevado ${ }^{6}$. El caso boliviano se encuentra por encima del promedio regional con un 33,3\% de gasto privado para la provisión del servicio educativo. Este análisis se revela aún más alarmante en el caso paraguayo: el componente privado asciende al 40,2\%, por encima no solo del promedio de OCDE, sino también del promedio latinoamericano.

No obstante, para completar el análisis de este indicador sería necesario valorar qué parte del gasto privado se dirige a la oferta educativa puramente privada y qué parte a la pública. Para este nivel de análisis tan específico, solo disponemos de datos comparables para Bolivia y Uruguay. Para el caso de Argentina se dispone de datos parciales, no estrictamente comparables, pero que nos permiten aproximarnos a la realidad. En este sentido, basándonos en el INEEd (2017), en Uruguay los hogares individuales e instituciones sin fines de lucro representan casi todo el gasto privado ( $24,7 \%)$, pero dicho porcentaje está destinado a financiar la prestación privada de la educación. Tan solo el $0,8 \%$ del gasto privado corresponde al esfuerzo de las familias dirigido al servicio público de educación. Sin embargo, en Bolivia, de acuerdo con UDAPE-UNICEF (2008), este tipo de gasto asciende al $17,4 \%$, dato que se encuentra por encima del ofrecido por Uruguay y que, además, es un 1,5\% más elevado que el gasto que las familias bolivianas destinan al sector privado de educación, lo que atañe directamente a la capacidad del Estado como provisor del servicio educativo ${ }^{7}$.

Tal como adelantábamos, en Argentina, aunque no es estrictamente comparable con la valoración previa, puede destacarse que "el 64\% del gasto que los hogares destinan a la educación corresponde a aranceles escolares privados" (Bottinelli y Sleiman, 2014: 4). Así pues, al menos el 8,3\% del total del gasto privado iría destinado a instituciones puramente privadas, por lo que la estructura del gasto privado se presume más equitativa que la boliviana y próxima a la uruguaya ${ }^{8}$. Ahora bien, según Centrágolo y Curcio (2017), para enfrentar la insuficiencia presupuestaria muchas instituciones educativas públicas requieren a las familias contribuciones extraordinarias que pueden pasar de una relativa voluntariedad a una obligatoriedad efectiva ${ }^{9}$.

Finalmente, para ofrecer una perspectiva completa del esfuerzo inversor en educación que realizan las familias se ha querido analizar el gasto promedio del hogar en educación como porcentaje del gasto total de los hogares. Así pues, en el extremo inferior se situaría Brasil con un porcentaje del 1,6\%. En una posición intermedia, con una proporción ligeramente inferior al promedio latinoamericano $(3,7 \%)^{10}$, se encuentran Argentina y Uruguay. Por encima del promedio regional está Paraguay (el gasto educativo de los hogares representa un 4,5\% del 
gasto total). En el extremo superior se sitúa Bolivia (este porcentaje asciende al 6,7\%), muy por encima del resto de países estudiados.

\section{Conclusiones}

En nuestro artículo nos preguntamos si la extensión de la obligatoriedad al nivel secundario de los sistemas escolares se puede interpretar como un reflejo de estos procesos de transferencia internacional y si ello guarda relación con las implicaciones efectivas que el derecho a la educación posee como derecho humano fundamental. El concepto de transferencia nos ha resultado útil en este estudio puesto que más que un proceso lineal y unidireccional lo hemos considerado multidimensional, es decir, aunque existen influencias externas en los procesos de reformas educativas, estas no son unidireccionales (cambian y se resignifican con los años) y adquieren además adaptaciones diferenciales en cada país y dentro de cada uno de ellos. Esto nos ha permitido estudiar las reformas educativas regionales en función de cómo estas avanzan y retroceden entre diferentes países y dentro de ellos, sobre todo en casos cuya organización institucional es federal y compleja (como Argentina o Brasil). Aunque este ejercicio intelectual referido a la transferencia de políticas de reformas también es pertinente en países de estructura territorial unitaria (Bolivia, Paraguay y Uruguay), ya que la aplicación de políticas y reformas trasvasa algunas de las características de los regímenes de gobierno. Un primer acercamiento a este análisis puede estar dado por uno de los conceptos más invocados en los procesos de reforma de la escuela secundaria obligatoria en la región: el derecho a la educación.

La configuración histórica del nivel secundario en la región ha contemplado mecanismos restrictivos de ingreso, y la permanencia y graduación de sus estudiantes ha evidenciado un aprovechamiento diferencial en función de los recursos y del capital social de los diferentes sectores sociales. Así, podríamos concluir que, si bien aumentó la cantidad de población escolarizada, ello no redundó necesariamente en un aumento equivalente en los niveles de graduación de la escuela secundaria. Podemos observar que en primaria el acceso se encuentra prácticamente garantizado en los países analizados, con una ligera reducción en Bolivia y Paraguay. Sin embargo, el acceso al nivel secundario se reduce en torno a un promedio del 10\% en Argentina, Bolivia y Brasil en comparación con el nivel primario, y alrededor de un 20\% en Paraguay y Uruguay. En estos dos últimos países los problemas de retención escolar y el rezago educativo se hacen más notorios. La comparación a través de los años permite destacar, en mayor medida, el esfuerzo realizado por Argentina y Uruguay, donde la tasa neta de acceso a secundaria ha aumentado en torno a un $10 \%$ promedio con respecto al año 2007.

Si se considera exclusivamente el nivel secundario de educación, la dispersión de los datos entre países es más evidente con respecto al nivel primario de educación. Esta tendencia también se nota en términos de permanencia y eficacia interna, ya que existen niveles bastante elevados de rezago escolar. De hecho, 3 de cada 10 estudiantes que asisten a la educación secundaria han repetido curso en 2 o más ocasiones en Argentina, Brasil y Uruguay, y 2 de cada 10 en Bolivia y Paraguay. El rezago existente afecta en menor medida a la permanencia escolar en Argentina y tiene mayor incidencia en países como Brasil, Bolivia y Uruguay. La situación más alarmante tiene lugar en Paraguay, donde 3 de cada 10 alumnos en edad de asistir a la educación secundaria superior no lo hacen. No obstante, este último indicador refleja no solo el abandono escolar que se produce en dicha etapa educativa, sino que también indica quienes ni siquiera llegaron a acceder a esta.

Igualmente, las cifras se vuelven mucho más desalentadoras al observar la conclusión de la educación secundaria en los países del Cono Sur estudiados. Solo Brasil presenta un escenario 
más positivo, dado que prácticamente 8 de cada 10 alumnos consiguen concluirla. Sin embargo, la finalización de la educación primaria está prácticamente universalizada. Si se examina la conclusión de ambos niveles combinados, podemos identificar diversos perfiles de países. En primer lugar, Brasil posee un alto egreso en el nivel primario y secundario. En segundo lugar, Argentina y Bolivia tienen un egreso alto en primaria y un egreso medio en secundaria. En tercer lugar, Paraguay y Uruguay tendrían un alto egreso en el nivel primario, pero bajo en el nivel secundario. En estos últimos solo 6 y 4 alumnos, respectivamente, consiguen culminar la educación secundaria seis años después de la edad de finalización teórica. Esta situación es alarmante en el caso uruguayo. A este respecto, según los estudios realizados por ANEP/TEMS (2000 y 2003) "el conjunto de expectativas, creencias $\mathrm{y}$ relaciones sociales que rodean al joven [...] son cruciales en el momento de tomar la decisión de desafiliación. También es relevante una vez más la historia escolar, específicamente la condición de repetidor" (Aristimuño, 2009: 189).

En suma, la extensión de la obligatoriedad será proporcional a la promoción del derecho a la educación en el nivel secundario en la medida en que sea acompañada de políticas públicas que contemplen modificaciones sustantivas de la retención y graduación, así como también medidas que garanticen una prestación aceptable y adaptable a las necesidades educativas de la población escolar en su conjunto. Esto podría suponer un camino insoslayable para la consecución de los objetivos derivados del derecho a la educación, en particular, de aquellos vinculados con la garantía de igualdad de oportunidades real para aquellos sectores de la población más vulnerables.

\section{Notas}

${ }^{1}$ El MERCOSUR se constituyó en el año 1991 y está integrado por Argentina, Brasil, Paraguay, Uruguay y Venezuela (que fue suspendido en diciembre de 2016). Bolivia es uno de los Estados asociados. Se trata de un acuerdo de unión aduanera (imperfecta) que prevé otras dimensiones de integración regional entre los Estados miembros. Entra esas dimensiones se encuentra la educativa.

2 Según Alexy (2008), la posición de derecho fundamental refiere a la capacidad o competencia jurídica para modificar una situación jurídica. Las posiciones tienen la propiedad de generar relaciones jurídicas entre los individuos, así como entre los individuos y el Estado.

${ }^{3}$ Diversos organismos internacionales, como la Organización de Estados Iberoamericanos (OEI) (2010, 2016) o la Comisión Económica para América Latina y el Caribe (CEPAL) (1997, 2010a, 2010b) o el Sistema de Información de Tendencias Educativas en América Latina (SITEAL) (2010), coinciden en señalar que son doce el mínimo de años de escolarización necesarios para evitar caer en la pobreza y garantizar el futuro laboral y social de los jóvenes, lo que coincide con la finalización de la educación secundaria.

${ }^{4}$ Se aclara que la falta de disponibilidad de datos oficiales actuales (sobre los indicadores incluidos en esta tabla) ha obligado a utilizar datos de diferentes fuentes y fechas, tal como se aclara en cada caso.

${ }^{5}$ Dato extraído de OEI (2016).

${ }^{6}$ Según la Organisation for Economic Co-operation and Development (OECD) (2017), el promedio de los países que forman parte de la OCDE es del 15,4\% para el año 2014.

${ }^{7} \mathrm{Si}$ bien es cierto, debemos tener cierta cautela en este último análisis dado que los datos relativos a Bolivia se basan a su vez en la encuesta de hogares del año 2005.

${ }^{8}$ Este $64 \%$ correspondería a gastos directos en educación privada, pero desconocemos a cuánto asciende el porcentaje relativo a los gastos indirectos o asociados. Así pues, el gasto de los hogares en el sector público todavía sería menor al 4,7\% del total del gasto privado. 


\footnotetext{
${ }^{9}$ En el caso argentino, el pago de dinero destinado a la asociación cooperadora de la escuela "es un ejemplo de cuota cuasi compulsiva" (Cetrángolo y Curcio, 2017: 50). En Bolivia, el pago de la matrícula y las pensiones ascendía a un $2,1 \%$. A este respecto, "aunque no deberían existir gastos directos en las escuelas públicas debido a la gratuidad de estas, existen y están registrados en la EH-2005. Esto podría reflejar el hecho de que algunas escuelas públicas incurren en cobros ilegales a los padres de familia" (UDAPE-UNICEF, 2008: 47).

${ }^{10}$ Dato extraído del Instituto Nacional de Evaluación Educativa (INEEd) (2017).
}

\section{Referencias bibliográficas}

Acerenza, S. y Gandelman, N. (2017). Household education spending in Latin America and the Caribbean: evidence from income and expenditure surveys. Montevideo: IDB.

Alexy, R. (2008). Teoría de los derechos fundamentales. Madrid: Centro de Estudios Políticos y Constitucionales.

Aristimuño, A. (2009). El abandono de los estudios del nivel medio en Uruguay: un problema complejo y persistente. Revista Iberoamericana sobre Calidad, Eficacia y Cambio en Educación, 7(4), 180-197. Recuperado de http://www.redalyc.org/articulo.oa?id=55114094010

Bottinelli, L. y Sleiman, C. (2014). ¿Cuánto gastan las familias en educación? Buenos Aires: UNIPE.

Caïs, J. (2002). Metodología del análisis comparativo. Madrid: Centro de Investigaciones Sociológicas.

CEPAL (1997). Panorama social de América Latina 1997. Santiago de Chile: Naciones Unidas.

CEPAL (2010a). Panorama social de América Latina 2010. Santiago de Chile: CEPAL.

CEPAL (2010b). El progreso de América Latina y el Caribe hacia los Objetivos de Desarrollo del Milenio. Desafíos para lograrlos con igualdad. Santiago de Chile: Naciones Unidas.

CEPAL (2018). CEPALSTAT. Base de datos y publicaciones estadísticas. Recuperado de http://estadisticas.cepal.org/cepalstat/Portada.html

Cetrángolo, O. y Curcio, J. (2017). Financiamiento y gasto educativo en América Latina. Santiago de Chile: CEPAL.

Gargarella, R. y Courtis, C. (2009). El nuevo constitucionalismo latinoamericano: promesas e interrogantes. Santiago de Chile: CEPAL.

INEEd (2017). Informe sobre el estado de la educación en Uruguay 2015-2016. Montevideo: INEEd.

Menezes-Filho, N. y Núñez, D. F. (2012). Estimando os gastos privados com educação no Brasil. Recuperado de https://www.yumpu.com/pt/document/view/12762615/estimando-os-gastos-privadoscom-educacao-no-brasil-insper

Molinier, L. (2016). El derecho a la educación y el gasto público en Paraguay. La importancia de los recursos del Fonacide y del Fondo para la Excelencia de la Educación y la Investigación (FEEI). Asunción: SERPAJ-PY.

OECD (2017). Education at a glance 2017: OECD indicators. París: OECD Publishing.

OEI (2010). Metas educativas 2021. La educación que queremos para la generación de los bicentenarios. Documento final. Madrid: OEI.

OEI (2016). Miradas sobre la Educación 2016. Avance en las Metas Educativas 2021. Madrid: OEI.

Roberston, S. y Dale, R. (2012). Investigación educativa en la era de la globalización. Más allá del nacionalismo metodológico, "educacionismo" metodológico y "fetichismo del espacio". En X. Rambla (ed.), La educación para todos en América Latina. Estudios sobre las desigualdades y la agenda política en educación (pp. 105-125). Buenos Aires: Miño y Dávila.

Schriewer, J. (2014). Neither orthodoxy nor randomness: differing logics of conducting comparative and international studies in education. Comparative Education, 50(1), 84-101. 
Scioscioli, S. (2014). El derecho a la educación como derecho fundamental y sus alcances en el derecho internacional de los derechos humanos. Journal of Supranational Policies of Education, 2, 6-24. Recuperado de https://revistas.uam.es/index.php/jospoe/article/view/5625/6039

SITEAL (2010). Informe sobre tendencias sociales y educativas en América Latina. Metas educativas 2021: desafíos y oportunidades. Buenos Aires: IPE-UNESCO-OEI.

SITEAL (2018). Base de datos. Recuperado de http://www.siteal.iipe.unesco.org/base_de_datos/consulta

Steiner-Khamsi, G. (2010). The politics and economics of comparison. Comparative Education Review 54(3), 323-342.

Tedesco, J. C. (2012). Educación y justicia social en América Latina. Buenos Aires: Fondo de Cultura Económica de Argentina.

Tomasevski, K. (2001). Human rights obligations: making education available, accessible, acceptable and adaptable. Estocolmo: Swedish International Development Cooperation Agency (SIDA). Recuperado de https://www.right-to-education.org/sites/right-to-education.org/files/resource-attachments/Tomasevski_Primer\%203.pdf

UDAPE/UNICEF (2008). El gasto de los hogares en educación. La Paz: UDAPE/UNICEF.

UNESCO (2018). Base de datos de la UNESCO-UIS.Stat. Recuperado de http://data.uis.unesco.org/

Urquiola, M. (2011). Calidad y cantidad educativa en Bolivia: 1996-2010. Informe preparado para el Ministerio de Relaciones Exteriores de Holanda. Recuperado de https://www.google.com/ search? client=safari $\&$ rls=en $\& \mathrm{q}=$ Calidad $+y+$ cantidad+educativa + en+Bolivia $+1996-2010 \& i e=U$ TF-8\&oe=UTF-8\#

Vila Lladosa, L. E. (2003). Los beneficios no monetarios de la educación. Revista de Educación, 331, 309-324.

\section{Abstract}

Secondary Education in five South American countries: normative definitions and empirical results from the right to education perspective

INTRODUCTION. The Latin American educational reforms have made normative definitions regarding the right to education with diverse objectives. We discuss the topics of the right to education and compulsory schooling since upper Secondary Education was made compulsory because of these reforms in five South American countries. RESULTS. The increase of compulsory education, including all secondary schooling, has been introduced by the governments as part of education reform policies. The main argument was that the compulsory secondary schooling would promote the right to education in these South American countries. METHOD. Data for this research has been collected from governmental agencies of the studied countries and it has included document analyses (legislation and curriculum designs). To analyze the data, we first create interim case summaries, documenting the case of each country and its education reform by drawing upon excerpts field notes. These memos were mainly descriptive, documenting the main topic that was object of reform, including data from investment in education and statistics of schooling. This method seeks to explain where a particular outcome varies across similar countries by identifying variables that account for this. The inclusion of the five cases only has a limited capacity in building a generalized explanation, but the variables (educational normative, educational statistics and investment) put forward in this article can serve as a basis for future applications on further sets of cases to advance the explanatory power of right to education. 
Thus, we track the recent trajectory of government policies for Secondary Education, which have been modified by these countries. DISCUSSION. We discuss the meaning of compulsory secondary education form a Human Rights based perspective, and the issues that are involved, such as: educational expansion and investment among countries.

Keywords: Right to education, Secondary school, Educational reforms, South America.

\section{Résumé}

L'enseignement secondaire dans cinq pays d'Amérique du Sud: définitions normatives et portée empirique du point de vue du droit à l'éducation

INTRODUCTION. En Amérique Latine, de nombreuses réformes de l'éducation ont progressé dans les normes normatives relatives au droit à l'éducation. On peut penser que ces réformes auront l'impact de la scène internationale des droits de l'homme et de l'intérieur des marchés nationaux. Dans cinq pays d'Amérique du Sud, l'extension des écoles obligatoires a été présentée comme une politique faisant partie intégrante du contenu de l'enseignement. MÉTHODE. En termes méthodologies, le desing de cette recherche comprend des techniques qualitatives, notamment une analyse juridique normative et la génération de typifications comparatives qui en découle, provenant des sources des pays considérés. Des techniques quantitatives sont également utilisées pour l'analyse des statistiques de l'éducation afin de pouvoir dimensionner la couverture éducative à la lumière des investissements budgétaires réalisés dans chaque État. RÉSULTATS. Dans ces pays, les réformes ont défini une nouvelle gamme d'études obligatoires englobant l'ensemble de l'enseignement secondaire. Parmi les arguments avancés, nous trouvons l'attention sur les exigences éducatives des secteurs qui n'appartenaient pas à l'école. Avec celà, il s'agit de promouvoir la réalisation d'une citoyenneté plus efficace chez les jeunes générations. DISCUSSION. Les concepts de droit à l'éducation et d'école obligatoire de la réforme dans cinq pays d'Amérique du Sud ont été redécrits car ils ont établi le caractère obligatoire de l'école secondaire. La comparaison transnationale se concentre sur les politiques de réforme de l'approche des droits de l'homme. Des articles tels que l'extension de l'obligation, la couverture et les investissements dans l'éducation sont analysés.

Mots-clés: Droit à l'éducation, Lycée, Réformes éducatives, Amérique du Sud.

\section{Perfil profesional de los autores}

\section{Guillermo Ramón Ruiz (autor de contacto)}

Es doctor en Ciencias de la Educación, profesor titular regular e investigador (equivalente a catedrático) en las facultades de Derecho y de Psicología de la Universidad de Buenos Aires e investigador del CONICET. Dirige proyectos I+D de la UBA y de la Agencia Nacional de Promoción de la Ciencia y la Tecnología. Sus actividades de investigación se centran en las reformas educativas de la educación secundaria, el debate pedagógico sobre el derecho a la educación y las políticas de formación de profesores en perspectiva internacional y comparada.

Correo electrónico de contacto: gruiz@derecho.uba.ar 
Dirección para la correspondencia: Avenida Figueroa Alcorta 2263 (C1425CKB), Ciudad Autónoma de Buenos Aires, Argentina.

\section{Sebastián Scioscioli}

Es doctor en Derecho. Profesor adjunto e investigador posdoctoral en la Facultad de Derecho de la Universidad de Buenos Aires. Es codirector de proyecto I+D de la Agencia Nacional de Promoción de la Ciencia y la Tecnología y ha sido investigador en varios proyectos de investigación de la UBA. Está especializado en temas de exigibilidad de los derechos sociales.

Correo electrónico de contacto: sebastianscioscioli@derecho.uba.ar

\section{Miriam Lorente Rodríguez}

Es doctora en Pedagogía. Profesora ayudante doctora en el Departamento de Educación Comparada e Historia de la Educación de la Universidad de Valencia. Imparte docencia en los Grados de Pedagogía, Educación Social y Magisterio de Educación Infantil. Es miembro investigador de proyectos de I+D de carácter internacional y nacional vinculados con el estudio del derecho a la educación en América Latina y a la profesionalización docente en España.

Correo electrónico de contacto: miriam.lorente@uv.es 